\title{
D-CRLH Based Band Rejection Filter using a Concavo-Convex Coupled CPW Transmission Line
}

\author{
Soo-Duk Seo*, Hak-Rae Cho* and Doo-Yeong Yang ${ }^{\dagger}$
}

\begin{abstract}
In this paper, the use of a dual composite right/left-handed coplanar waveguide (CPW) transmission line is proposed for the design of a band rejection filter. The notch property of the filter is achieved by combining the convex signal line with the shorted concave meander line, and the equivalent circuit model is extracted from the geometry of the unit cell for organizing the band rejection property. Then the equivalent parameters of the unit cell are analyzed to identify those behaviors. And the dispersion characteristics and energy distributions are simulated. In the end, the band rejection filter is manufactured by cascading two proposed unit cells. We show that the measurement result for the resonant frequency demonstrates good agreement with the simulation result and the band rejection filter provides a rejection performance of $17.5 \mathrm{~dB}$ at the stopband frequency ranging from $869 \mathrm{MHz}$ to $894 \mathrm{MHz}$.
\end{abstract}

Keywords: D-CRLH, Band rejection filter, Notch property, Convex signal line, Shorted concave meander line

\section{Introduction}

Over the past decade, metamaterials (MTMs) with simultaneously negative values of $\varepsilon$ and $\mu$ have received extensive attention in many areas. Special properties of this material were demonstrated experimentally in [1] and [2]. After that, a left-handed (LH) transmission line approach was introduced. This approach was applied to the efficient design of microwave applications owing to the wide bandwidth, low loss and size miniaturization. The conventional structure for the LH transmission line is called a conventional composite right/left-handed (CCRLH) structure [3-7], which has both a LH range due to the effect of the loading elements and a right-handed (RH) range due to the parasitic elements of the transmission line. On the other hand, there is a dual structure of C-CRLH known as a dual composite right/left-handed (D-CRLH) [8-13]. The D-CRLH transmission line exhibits a RH passband at low frequencies and a LH passband at high frequencies, which correspond to the band rejection property.

A coplanar waveguide transmission line (CPW TL), utilized to compose the D-CRLH property, has been widely used in microwave integrated circuits (MIC) and monolithic microwave integrated circuit (MMIC) since the signal line and ground planes are placed on the same layer, which makes it easy to facilitate shunt connections and fabrication $[14,15]$.

$\dagger$ Corresponding Author: Dept. of Telecommunication Engineering, Jeju National University, Korea. (yeongyd@jejunu.ac.kr)

* Innertron R\&D Lab, Innertron Inc., Incheon, Korea.

(sds@ innertron.co.kr, happycho@innertron.co.kr)

Received: August 7, 2014; Accepted: November 1, 2015
In this paper, a unit cell with D-CRLH band rejection property on the CPW TL is investigated. In order to obtain the band rejection property, the concave meander line, coupled with the convex signal line, is connected in the metallic ground plane of CPW TL. From this configuration, the equivalent circuit model for the unit cell is extracted and analyzed. The behaviors of the parameters in the unit cell are simulated using the high frequency structural simulator (HFSS) [16]. Additionally, the dispersion characteristics and energy distribution of the electromagnetic field are simulated to validate the property of the unit cell. Finally, the band rejection filter is designed and fabricated by cascading the proposed unit cells and the $\mathrm{S}$ parameters of the filter are measured by a vector network analyzer (Agilent N5230A). The simulation results are then compared with the measurement results.

\section{Unit Cell Based on D-CRLH CPW TL}

Fig. 1 shows the physical geometry of the proposed unit cell based on the modified D-CRLH with a concave meander line connected on the ground plane of CPW. The unit cell is designed on the substrate with a thickness of $\mathrm{H}$ $=0.787 \mathrm{~mm}$, a dielectric permittivity of $\varepsilon_{r}=2.5$ and a conductor thickness $T_{c}$ of $35 \mu \mathrm{m}$. Moreover, the input and output ports of the unit cell are set to a $50 \Omega$ characteristic impedance in the CPW configuration, which results in a feeder line width $\mathrm{W}=8.74 \mathrm{~mm}$ and the gap width between the feeder line and the side ground planes being $G=0.3$ $\mathrm{mm}$.

The parallel coupled CPW transmission line can be used to construct many types of filters. In particular, a coupled 
line band rejection filter can be easily fabricated using a CPW TL, for bandwidths less than $20 \%$. In this study, the coupled line filter is implemented and combined a convex signal line with a shorted concave meander line section whose length is a quarter-wavelength of the notch center frequency.

The concave meander line is placed on a side ground plane of CPW TL. One side of the meander line is shorted to the adjacent ground plane and the other is opened. In order to improve the coupling effect between the convex signal line and the concave meander line, the geometry of the proposed unit cell is constructed as Fig. 1. The narrowband band rejection filter can be made with the cascaded coupled line sections shown in Fig. 1. In an effectively homogeneous structure defined as electromagnetic MTMs, the physical length $l$ of a conventional D-CRLH geometry is required to be smaller than one fourth of the guided wavelength. Thus, the length $l$ of the proposed unit cell should be smaller than a quarter-wave length, at the least.

To drive the design equations for this type of filter, we first show that a single coupled line section can be approximately modeled by the equivalent circuit shown in Fig. 2(a).

$$
\begin{gathered}
Z_{\text {in } 1}=\frac{2 Z_{0 e} Z_{0 o} \cos \theta}{\sqrt{\left(Z_{0 e}+Z_{0 o}\right)^{2} \cos ^{2} \theta-\left(Z_{0 e}-Z_{0 o}\right)^{2}}} \\
Z_{i n 2}=\frac{Z_{0 e} Z_{0 o}}{Z_{i n 1}}
\end{gathered}
$$

$\mathrm{Z}_{\text {in1 }}$ and $\mathrm{Z}_{\text {in2 }}$ are the input impedances at port 1 and 2 [17].

$\mathrm{Z}_{0 \mathrm{e}}$ and $\mathrm{Z}_{0 \mathrm{o}}$ are the characteristics impedance of the parallel coupled CPW TL in even and odd mode $[15,18]$. $\theta$ is the electrical length of the coupled line section with $2 \pi l / \lambda$. The length of the coupled line section in (1) is equal to approximately $\theta=\pi / 2$ and $l=\lambda / 4$, which corresponds to the center frequency of the band rejection response. In this case, as the $Z_{\text {in } 1}=\infty, Z_{\text {in } 2}=0$, input signal at the port 1 does not transmit to port 2 and the signal at port 2 does not couple to the meander line. The band rejection characteristics occur when the electrical length of the coupled meander line is a quarter-wave length. However, at the other frequencies, the input signal transmits into the output port without attenuation.

For a low loss CPW TL, the coupling model for the unit cell shown in Fig. 1 consists of both a series-connected parallel resonant circuit and a shunt-connected parallel resonant circuit, as shown in Fig. 2 (a). As this resonant configuration contributes an additional coupling capacitance component to the property of the convex signal line, it can be modeled as a series RLC circuit of parallel resonance. Also, as the shorted concave meander line is set to a quarter of a wavelength for the notch frequency, it can be equivalently modeled as a shunt GLC circuit of parallel resonance. Therefore, the equivalent circuit for the unit cell

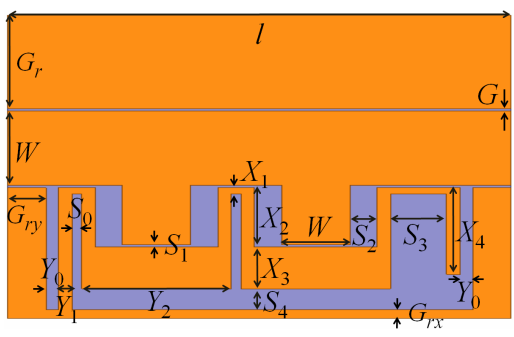

Fig. 1. Physical geometry of the unit cell

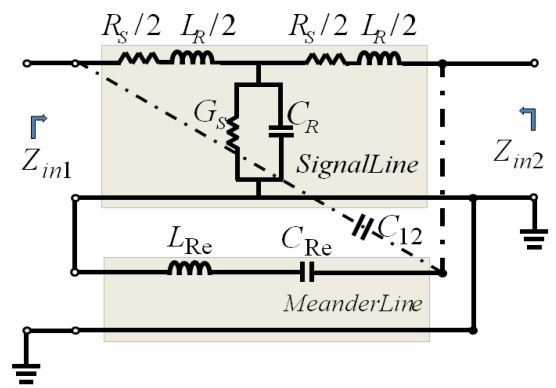

(a)

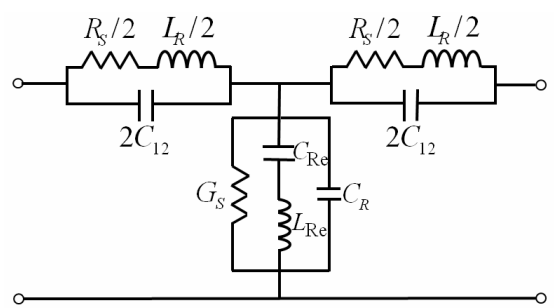

(b)

Fig. 2. Equivalent circuit for the unit cell (a) coupling model and (b) simplified model

can be represented as shown in Fig. 2 (b). In the equivalent circuit components using a low-loss CPW TL, the coupling capacitance $\mathrm{C}_{12}$ of the series-connected RLC parallel resonant circuit depends on the coupling gap between the convex signal line and the shorted concave meander line. In addition, the inductance $\mathrm{L}_{\mathrm{R}}$ and the capacitance $\mathrm{C}_{\mathrm{R}}$ mainly depend on the length and width of the convex signal line. In particular, the inductance $\mathrm{L}_{\mathrm{Re}}$ and capacitance $\mathrm{C}_{\mathrm{Re}}$ of the shunt-connected GLC shunt resonant circuit rely on the dimensions of the shorted meander line and play an important role in the band rejection property. The $R_{S}$ and $\mathrm{G}_{\mathrm{S}}$ depend intimately on the conductivity of the conductor and dielectric substrate of CPW TL, respectively.

Consequently, the equivalent impedance $\mathrm{Z}$, admittance $\mathrm{Y}$, the series resonant frequency $\omega_{\text {se }}$ of the coupled CPW TL and the shunt resonant frequency $\omega_{\text {sh }}$ of the meander line can be formulated as follows:

$$
\begin{gathered}
Z=\frac{\left(R_{S}+j \omega L_{R}\right)\left\{1-\left(\omega / \omega_{s e}\right)^{2}-j \omega R_{S} C_{12}\right\}}{\left\{1-\left(\omega / \omega_{s e}\right)^{2}\right\}^{2}+\left(\omega R_{S} C_{12}\right)^{2}} \\
Y=\frac{G_{S}\left\{1-\left(\omega / \omega_{s h}\right)^{2}\right\}+j \omega\left[C_{\operatorname{Re}}+C_{R}\left\{1-\left(\omega / \omega_{s h}\right)^{2}\right\}\right]}{1-\left(\omega / \omega_{s h}\right)^{2}}
\end{gathered}
$$




$$
\omega_{s e}=\frac{1}{\sqrt{L_{R} C_{12}}}, \omega_{s h}=\frac{1}{\sqrt{L_{\mathrm{Re}} C_{\mathrm{Re}}}}
$$

The propagation constant, attenuation constant and phase constant of the equivalent circuit model can be calculated from the transmission parameter of $S_{21}$.

$$
\begin{aligned}
\gamma & =\alpha+j \beta=\sqrt{Z Y} \\
& =-\frac{1}{l}\left[\ln \left(\left|S_{21}\right|\right)+j \phi\left(S_{21}\right)\right]
\end{aligned}
$$

The dispersion characteristics of $\beta$ generally appear as a continuous function of frequency in the form of a curve varying within $|\beta| \leq \pi$. The 1 and $\phi\left(S_{21}\right)$ represent the physical length of the unit cell in a specific implementation and the phase of $S_{21}$, respectively.

\section{Simulation Results of the Unit Cell}

To investigate the sensitivities of the parameters in the unit cell, the main parameters $S_{0}, S_{1}, X_{2}$ and $Y_{2}$ are chosen to test. The insertion loss characteristics for the variation of the main parameters are shown in Fig. 3. When $S_{0}$ and $Y_{2}$ are increased, the length of the concave meander line grows longer and the coupling area becomes wider. As a result, the resonant frequency decreases as shown in Fig. 3(a) and (b). It can be denoted as the values of $\mathrm{L}_{\mathrm{Re}}$ and $\mathrm{C}_{\mathrm{Re}}$ increasing. Meanwhile, based on widening the coupling gap $S_{1}$, the resonant frequency increases as shown in Fig. 3(c) since the weakened coupling resonant effect decreases the $\mathrm{C}_{12}$ component. In addition, the resonant frequency tends to go to lower frequencies as shown in Fig. 3(d) when $\mathrm{X}_{2}$ increases. The reason why $\mathrm{L}_{\mathrm{Re}}$ and $\mathrm{C}_{\mathrm{Re}}$ have larger values is that the coupling area and the length of the meander line grow larger. The equivalent immittance parameters referenced in [14-16] are $\mathrm{R}_{\mathrm{S}}=0.54 \Omega, \mathrm{L}_{\mathrm{R}}=2.6$ $\mathrm{nH}, \mathrm{C}_{\mathrm{R}}=1.02 \mathrm{pF}, \mathrm{C}_{12}=0.14 \mathrm{pF}, \mathrm{G}_{\mathrm{S}}=0.001 \mathrm{~S}, \mathrm{~L}_{\mathrm{Re}}=12.58$ $\mathrm{nH}$ and $\mathrm{C}_{\mathrm{Re}}=2.55 \mathrm{pF}$, respectively.

Fig. 4 shows the dispersion characteristics of the unit cell, which were obtained through HFSS using S-parameter values. It can be seen that the unit cell exhibits a lowfrequency $\mathrm{RH}$ passband from $\mathrm{DC}$ to $824 \mathrm{MHz}$, a midfrequency stopband from $869 \mathrm{MHz}$ to $894 \mathrm{MHz}$ and a highfrequency RH passband above $895 \mathrm{MHz}$. The LH passband exists in the intermediate frequency range of $824 \mathrm{MHz}$ to $869 \mathrm{MHz}$. Therefore, the proposed unit cell includes DCRLH characteristics.

The energy distribution of electric fields is shown in Fig. 5(a), corresponding to the accumulated energy distribution of the capacitance $\mathrm{C}_{12}$ generated in proportion to the coupling gap between the convex signal line and the shorted concave meander line. As time elapses, the electric energy turns out to be the magnetic energy and the magnetic energy distribution corresponds to the

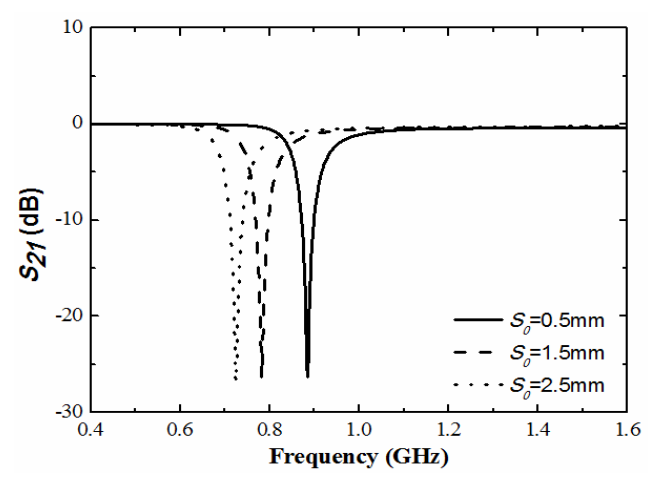

(a)

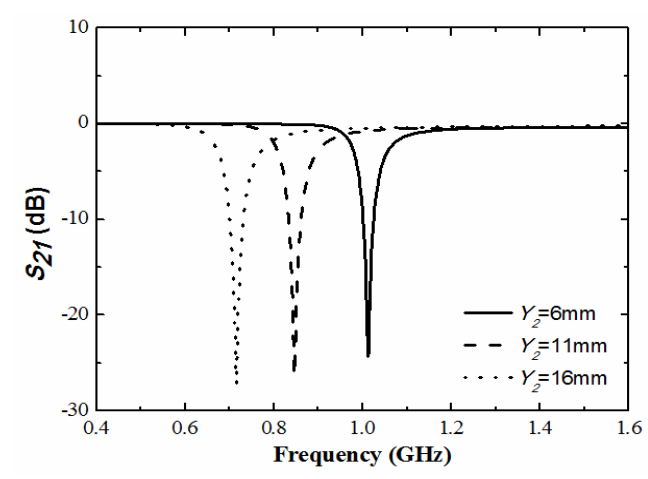

(b)

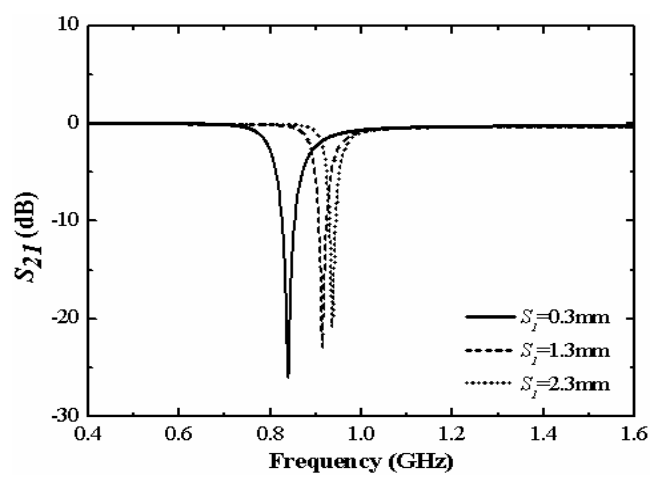

(c)

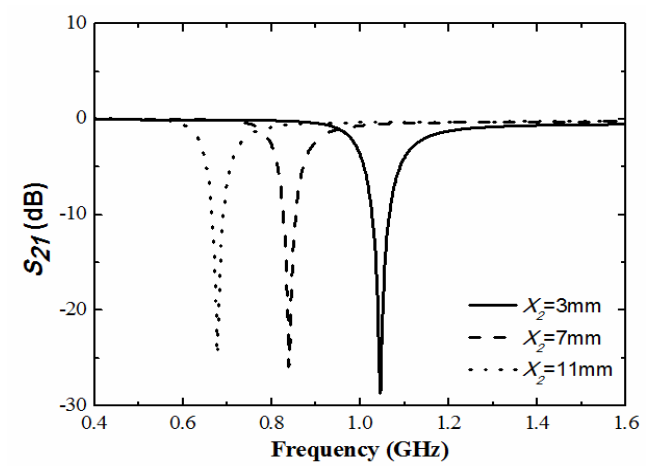

(d)

Fig. 3. $S_{21}(d B)$ characteristics as a function of geometrical parameters: (a) $S_{0}$, (b) $Y_{2}$, (c) $S_{1}$ and (d) $X_{2}$ 


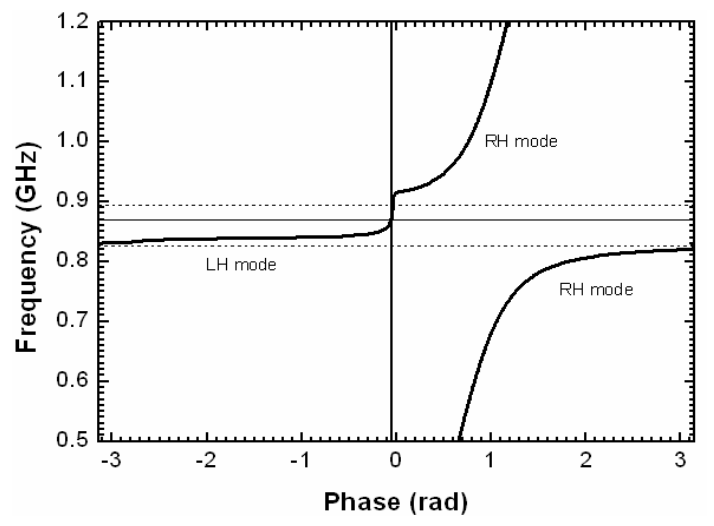

Fig. 4. Dispersion characteristics of the unit cell

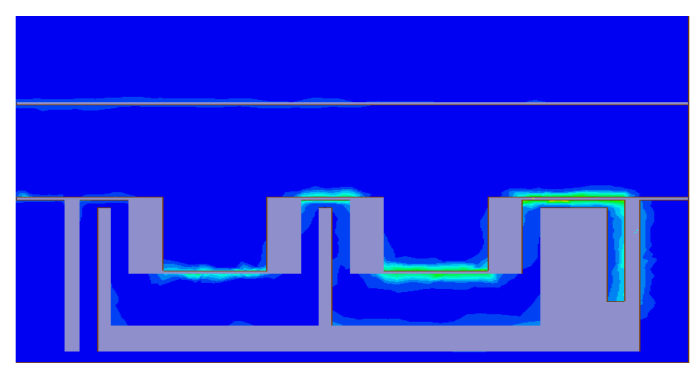

(a)

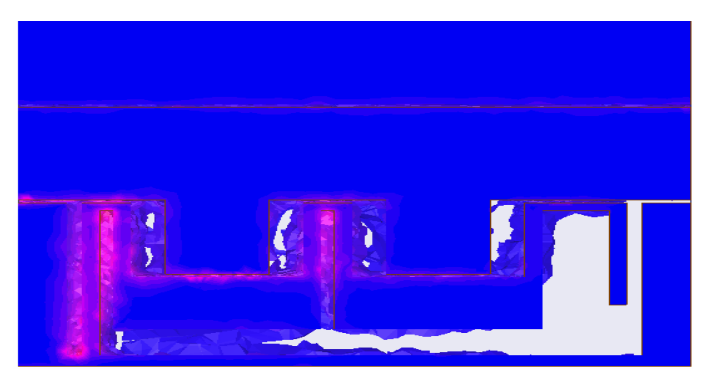

(b)

Fig. 5. Energy distributions of the electromagnetic field: (a) Electric energy and (b) magnetic energy

accumulated energy distribution of the inductance $\mathrm{L}_{\mathrm{Re}}$, which is distributed on the shorted concave meander line. This process is repeated while the signal energy transmits from the input port to the output port. Eventually, the unit cell obtains the characteristics of preserving the input electromagnetic energy and transmitting the energy to the output port with a small loss, as well as band rejection frequency selectivity.

The unit cell is fabricated in dimensions in Table 1 and the measurement results are obtained with an Agilent N5230A vector network analyzer. A comparison of the Sparameter simulation and measured results is provided in Fig. 6. It shows that the measurement results have good agreement with the simulation results regarding resonant frequency. The simulated resonant frequency of the unit cell is $881 \mathrm{MHz}$ and the measured resonant frequency is $880 \mathrm{MHz}$, with an error of $0.11 \%$ at the most. The
Table 1. The physical dimensions of the unit cell

\begin{tabular}{c|c|c|c|c|c}
\hline Parameters & $\mathrm{G}_{\mathrm{r}}$ & $\mathrm{L}$ & $\mathrm{G}$ & $\mathrm{W}$ & $\mathrm{G}_{\mathrm{rx}}$ \\
\hline Values $(\mathrm{mm})$ & 10 & 36.9 & 0.3 & 8.74 & 1 \\
\hline Parameters & $\mathrm{G}_{\mathrm{ry}}$ & $\mathrm{X}_{1}$ & $\mathrm{X}_{2}$ & $\mathrm{X}_{3}$ & $\mathrm{X}_{4}$ \\
\hline Values $(\mathrm{mm})$ & 2.8 & 0.7 & 7 & 4.9 & 9.6 \\
\hline Parameters & $\mathrm{Y}_{0}$ & $\mathrm{Y}_{1}$ & $\mathrm{Y}_{2}$ & $\mathrm{~S}_{0}$ & $\mathrm{~S}_{1}$ \\
\hline Values $(\mathrm{mm})$ & 4 & 1 & 11 & 0.8 & 0.3 \\
\hline Parameters & $\mathrm{S}_{2}$ & $\mathrm{~S}_{3}$ & $\mathrm{~S}_{4}$ & & \\
\hline Values $(\mathrm{mm})$ & 2 & 2.5 & 0.9 & & \\
\hline
\end{tabular}

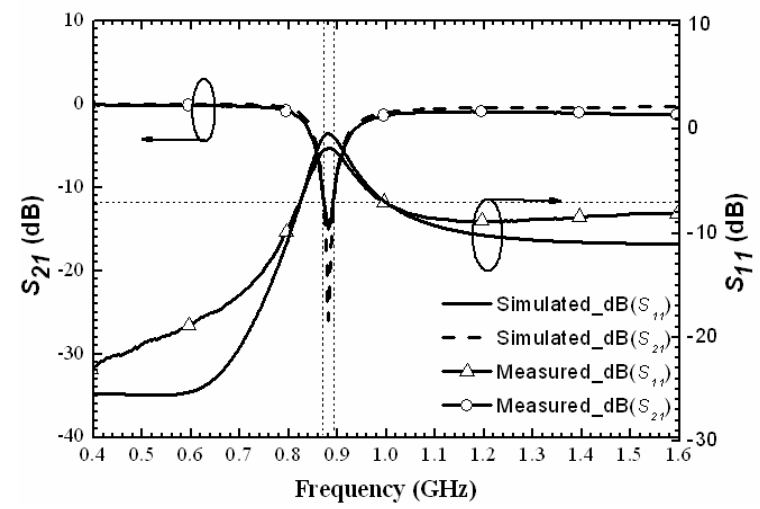

Fig. 6. S-parameter simulation and measurement results of the unit cell

measurement shows that the unit cell has a small return loss of less than $2 \mathrm{~dB}$ and a band rejection property of 12 $\mathrm{dB}$ in the stopband from $869 \mathrm{MHz}$ to $894 \mathrm{MHz}$.

\section{Measurement Results of the Band Rejection Filter}

Based on the properties discussed above, the proposed unit cell can be used for band rejection filter design. In order to obtain the desired passband and sufficient suppression in the stopband, two unit cells are adjusted by $\mathrm{X}_{4}$ and cascaded in a series through the separation of $\mathrm{S}_{5}$.

Fig. 7 shows the effect of the variation of the $S_{5}$ and $X_{4}$ parameter. As shown in Fig. 7 (a), the $S_{5}$ has to be separated at a far enough distance to obtain the desired bandwidth and band rejection. When the gap distance $S_{5}$ is less than $10.6 \mathrm{~mm}$, the parasitic coupled capacitance coupled between the two shorted meander lines grows larger. The capacitance changes to resonant frequency and rejection bandwidth, creating bad effects. In addition, after adjusting the total effective length of the shorted meander line to a quarter- wavelength and according to the variation of the length of $\mathrm{X}_{4}$, the rejection performance and resonance frequency of the filter can be adjusted in detail, as shown in Fig. 7 (b). The notch bandwidth and band rejection performance are achieved very well when the $\mathrm{S}_{5}$ and $\mathrm{X}_{4}$ set to $8.9 \mathrm{~mm}$.

Fig. 8 shows the physical geometry of the proposed band rejection filter. The dimensions are shown in Table 2. 
Table 2. Physical dimensions of the band rejection filter

\begin{tabular}{c|c|c|c|c|c}
\hline Parameters & $\mathrm{G}_{\mathrm{r}}$ & $\mathrm{L}$ & $\mathrm{G}$ & $\mathrm{W}$ & $\mathrm{G}_{\mathrm{rx}}$ \\
\hline Values $(\mathrm{mm})$ & 10 & 75.8 & 0.3 & 8.74 & 1 \\
\hline Parameters & $\mathrm{G}_{\mathrm{ry}}$ & $\mathrm{X}_{1}$ & $\mathrm{X}_{2}$ & $\mathrm{X}_{3}$ & $\mathrm{X}_{4}$ \\
\hline Values $(\mathrm{mm})$ & 2.8 & 0.7 & 7 & 4.9 & 8.9 \\
\hline Parameters & $\mathrm{Y}_{0}$ & $\mathrm{Y}_{1}$ & $\mathrm{Y}_{2}$ & $\mathrm{~S}_{0}$ & $\mathrm{~S}_{1}$ \\
\hline Values $(\mathrm{mm})$ & 4 & 1 & 11 & 0.8 & 0.3 \\
\hline Parameters & $\mathrm{S}_{2}$ & $\mathrm{~S}_{3}$ & $\mathrm{~S}_{4}$ & $\mathrm{~S}_{5}$ & \\
\hline Values $(\mathrm{mm})$ & 0.9 & 4.1 & 0.9 & 8.9 & \\
\hline
\end{tabular}

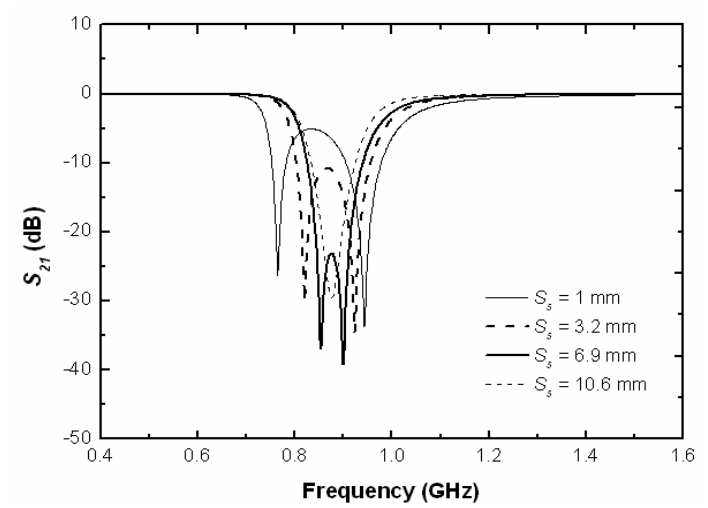

(a)

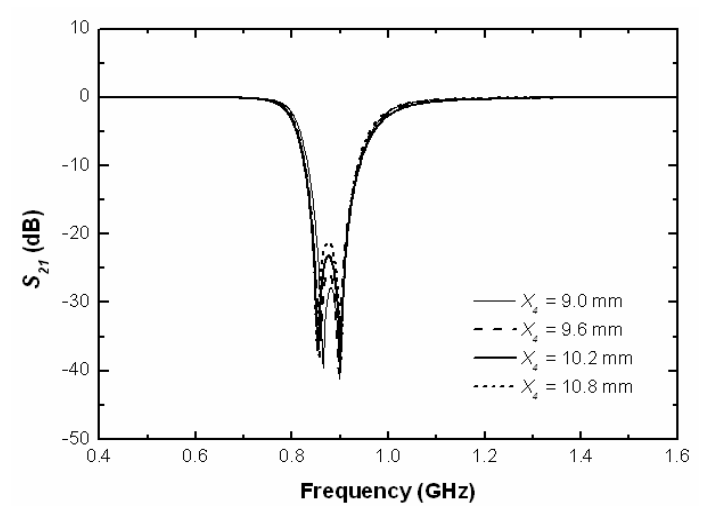

(b)

Fig. 7. $\mathrm{S}_{21}(\mathrm{~dB})$ characteristics of the band rejection filter as a function of geometrical parameters: (a) $\mathrm{S}_{5}$, and (b) $\mathrm{X}_{4}$.

A comparison of the simulation and experimental results is given in Fig. 9, which shows good agreement from the point of view of the resonant frequency of 880 $\mathrm{MHz}$. The measured result shows the band rejection of $17.5 \mathrm{~dB}$ at the stopband from $869 \mathrm{MHz}$ to $894 \mathrm{MHz}$. In practical implementation, the suppression performance over the stopband didn't constitute a sufficient condition as well as the simulation results because of the dispersive and parasitic loss of the CPW TL.

\section{Conclusion}

A band rejection filter has been realized with cascading

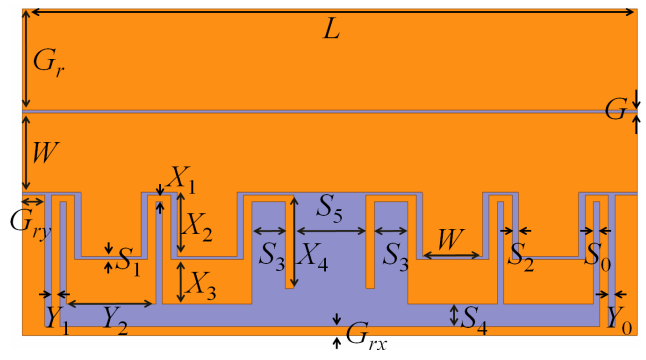

(a)

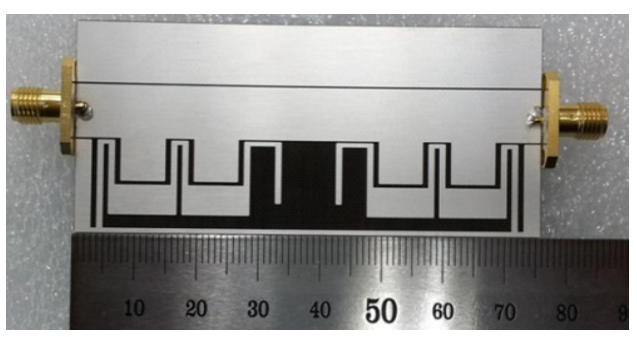

(b)

Fig. 8. Physical geometry of the proposed band rejection filter: (a) Simulated layout pattern and (b) fabricated layout pattern

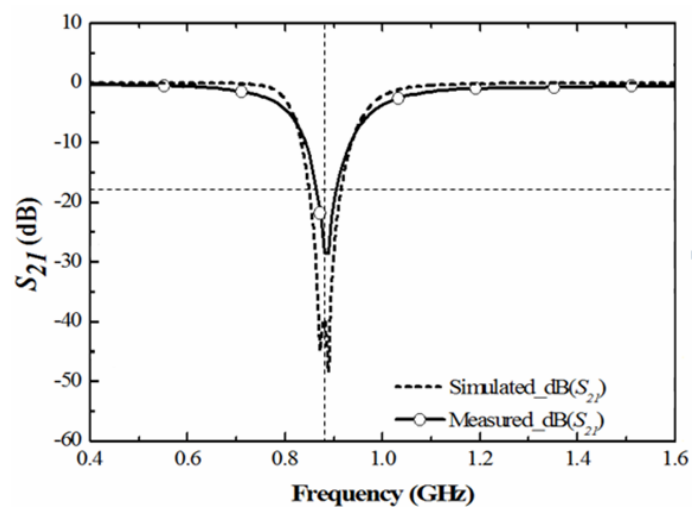

Fig. 9. S-parameter simulation and measurement results of the band rejection filter

unit cells based on the D-CRLH transmission line theory. The physical geometry of the unit cell, which combines the convex signal line with the shorted concave meander line, is presented. The equivalent circuit model for the proposed unit cell has been constructed from the simulation results of its design parameters. The modified model, based on the D-CRLH CPW transmission line, consists of a series RLC circuit of parallel resonance and a shunt GLC circuit of parallel resonance. From the dispersion diagram, the unit cell exhibits a low-frequency $\mathrm{RH}$ passband in DC-824 $\mathrm{MHz}$, an intermediate $\mathrm{LH}$ passband in the range of 825 to $868 \mathrm{MHz}$, a mid-frequency stopband in the range of 869 to $894 \mathrm{MHz}$ and a high-frequency RH passband above 895 MHz. As a result, the band rejection filter has been fabricated by cascading two proposed unit cells. We show that the filter has a band rejection property of $17.5 \mathrm{~dB}$ in 
the stopband frequency range of $869 \mathrm{MHz}$ to $894 \mathrm{MHz}$.

\section{Acknowledgements}

This research was supported by the Advanced Technology Center (ATC) funded by Ministry of Knowledge Economy, Korea.

\section{References}

[1] V. Veselago, "The Electrodynamics of Substances with Simultaneously Negative Values of $\varepsilon$ and $\mu$," Soviet Physics Uspekhi, vol. 10, no. 4, Feb. pp. 509514, 1968.

[2] D. R. Smith, W. J. Padilla, D. C. Vier, S. C. NematNasser and S. Schultz, "Composite Medium with Simultaneously Negative Permeability and Permittivity," Phys. Rev. Lett., vol. 84, no. 18, pp. 4184-4187, May 2000.

[3] C. Caloz and T. Itoh, Electromagnetic Metamaterials: Transmission Line Theory and Microwave Applications, John Wiley \& Sons, Inc., p. 15, 2006.

[4] C. Caloz and T. Itoh, "Novel Microwave Devices and Structures Based on the Transmission Line Approach of Meta-materials," Microwave Symposium Digest, IEEE MTT-S International, vol. 1, pp. 195-198, June 2003.

[5] A. Lai, T. Itoh and C. Caloz, "Composite Right/LeftHanded Transmission Line Metamaterials," IEEE Microwave Magazine, pp. 34-50, Sept. 2004.

[6] F. J. Herraiz-Martinez, V. Gonzalez-Posadas, F. Inigo-Villacorta and D. Segovia-Vargas, "Low Cost Approach Based on an Eigen-frequency Method to Obtain the Dispersion Diagram in CRLH Structure," IEEE Microwave Wireless Component Lett, vol. 17, no.1, pp.13-15, 2007.

[7] Qi Zhu and Shan-Jia Xu, "Composite Right/Left Handed Transmission Line Metamaterials and Applications," Meta. 2008 International workshop, pp. 7275, Nov. 2008.

[8] C. Caloz, "Dual Composite Right/Left-handed (DCRLH) Transmission Line Metamaterial," IEEE Microw. Wireless Component Lett., vol. 16, no. 11, pp. 585-587, Nov. 2006.

[9] X. Hu, P. Zhang and S. He, "Dual Structure of Composite Right/Left Handed Transmission Line," Proc. Biophotonics, Nonophotonics and Materials, Rome, pp. 529-532, Oct. 2006.

[10] X. Hu, P. Zhang, "A Novel Dual-band Balun Based on the Dual Structure of Composite Right/Left Handed Transmission Line," J. Zhejiang Univ. Sci. A, vol. 1, pp. 529-532, 2006.

[11] W. Tong, Z. Hu, H. Zhang, C. Caloz and A. Rennings, "Study and Realization of Dual-composite Right/
Left-Handed Coplanar Waveguide Metameterial in MMIC Technology," IET Microwave Antennas Propagation, vol. 2, no. 7, pp. 731-736, 2008.

[12] V. Gonzalez-Posadas, J. L. Jimenez-Martin, A. ParraCerrada, L. E. Garcia-Munoz and D. Segovia-Vargas, "Dual-composite Right-left-handed Transmission Lines for the Design of Compact Diplexers," IET Microwave Antennas Propagation, vol. 4, no. 8, pp. 982-990, 2010.

[13] H. Lee and T. Itoh, "Isolation Circuits Based on Metamaterial Transmission Lines for Multiplexer," Journal of Electromagnetic Engineering and Science, vol. 13, no.3, pp. 141-150, 2013.

[14] Cheng P. Wen, "Coplanar Waveguide: a Surface Strip Transmission Line Suitable for Nonreciprocal Gyromagnetic Device Applications," IEEE Transactions on Microwave Theory and Techniques, vol. 17, no. 12, pp. 1087-1090, Dec. 1969.

[15] Rainee N. Simons, Coplanar Waveguide Circuits, Components, and Systems, John Wiley \& Sons, Inc., pp. 11-86, 171-285, 2001.

[16] A. Lai, Left-handed Metameterials for Microwave Engineering Applications, Application Workshops for High-performance Electronic Design Guide, Ansoft HFSS.

[17] David M. Pozar, Microwave Engineering $2^{\text {nd }}$ Ed., John Wiley \& Sons, Inc., pp. 56-103, 474-478, 1998.

[18] S. S. Bedair and I. Wolff, "Fast Accurate and Simple Approximate Analytic Formulas for Calculating the Parameters of Supported Coplanar Waveguides for (M)MIC's," IEEE Transactions on Microwave Theory and Techniques, vol. 40, no. 1, pp. 41-48, 1992.

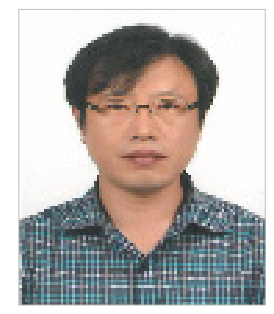

Soo-Duk Seo $\mathrm{He}$ received B.S and M.S in telecommunication engineering from Jeju National University, Korea in 1996, 1998, respectively. And then, He worked for eight years at R\&D Research Institute of Ace Technology in Korea. Since 2007, He has been a R\&D chief of Research Institute of Innertron, Inc. in Korea. His main research interests include RF devices and wireless communication systems.

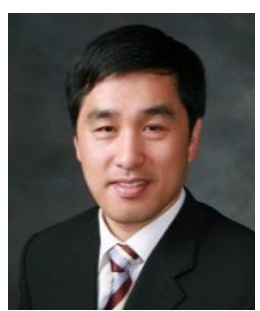

Hak-Rae Cho He received B.S in material engineering from Kyungnam University, Korea in 1998. And then, $\mathrm{He}$ worked for one year at Ace Technology in Korea. Since 2002, He has been a president of Innertron, Inc. in Korea. His main research interests include RF \& wireless communication systems and management information systems. 


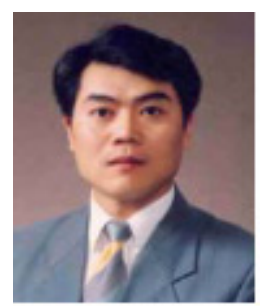

Doo-Yeong Yang He received B.S degree in telecommunication engineering from Jeju National University, Korea in 1984, and M.S, Ph.D degrees in electronic and telecommunication engineering from Hanyang University, Korea in 1989, 1992 respectively. Since 1992, he has been a professor in telecommunication engineering of Jeju National University in Korea. His current research interests include RF devices, microwave circuits and wireless and satellite communication systems. 